\section{Influencia del polimorfismo de la enzima convertidora de angiotensina 2 (rs1978124) en la respuesta hemodinámica posterior a la prueba aeróbica submáxima en jóvenes estudiantes chilenos sedentarios}

\author{
NACIM MOLINA GUTIÉRREZ ${ }^{1, a}$, SANDRA BECERRA MUÑOZ ${ }^{2, b}$, \\ ALEJANDRO PACHECO VALLES ${ }^{3, c}$
}

\section{Effect of angiotensin converting enzyme 2 rs1978124 gene polymorphism on the hemodynamic response to exercise}

Background: Angiotensin-converting enzyme 2 (ACE2) has a protective role in the regulation of blood pressure. Aim: To evaluate the influence of ACE2 rs1978124 gene polymorphism on the hemodynamic response after a six-minute walk submaximal aerobic test. Material and Methods: A six-minute walk submaximal aerobic test was carried out in 47 men and 55 women, aged $22 \pm$ 2 years. Blood pressure was recorded before and after the test. Pulse rate was recorded continuously. ACE2 polymorphism was determined in DNA extracted from a blood sample. Results: For women, the genotype distribution did not deviate from Hardy-Weinberg equilibrium $\left(\chi^{2}=0.804\right.$, d.f. $\left.=1, P=0.4\right)$ and no significant differences in heart rate, systolic and diastolic blood pressure were observed between CC, CT and TT genotypes before and after the test. Among men no differences in these parameters were observed either between the three phenotypes. Conclusions: ACE2 rs1978124 gene polymorphism did not influence the hemodynamic response to submaximal exercise in these participants.

(Rev Med Chile 2021; 149: 733-737)

Key words: Exercise; Hemodynamics; Polymorphism, Single Nucleotide.
'Universidad Católica del Maule, Facultad de Ciencias de la Salud, Departamento de Kinesiología. Talca, Chile.

${ }^{2}$ Universidad de Talca, Facultad de Ciencias de la Salud, Escuela de Kinesiología. Talca, Chile. ${ }^{3}$ Universidad Católica del Maule, Facultad de Ciencias de la Salud, Departamento de Kinesiología.

Talca, Chile.

aProfesor de Educación Física; Kinesiólogo; Magister en Kinesiología ${ }^{\circ}$

'Kinesióloga, Magister en Kinesiología.

'Tecnólogo Médico, PhD en

Biología Celular y Molecular.

Apoyo Financiero: Los autores reconocen el generoso apoyo a este proyecto proporcionado por el Departamento de Kinesiología de la Universidad Católica del Maule, quienes aportaron con laboratorios y equipos que permitieron realizar este estudio.

Los autores declaran no tener conflictos de interés.

Recibido el 10 de mayo de 2020, aceptado el 25 de mayo de 2021

Correspondencia a

Alejandro Pacheco Valles Universidad Católica del Maule, Facultad de Ciencias de la Salud, Departamento de Kinesiología, Talca, Chile. Avda. San Miguel 3605. Talca, Chile. apacheco@ucm.cl
L a condición física es la capacidad del cuerpo para realizar un esfuerzo físico pesado por un largo período de tiempo utilizando grandes grupos de músculos sin algún desequilibrio significativo de la homeostasis ${ }^{1}$. La presión arterial (PA) se eleva en respuesta al ejercicio físico y como sabemos, la magnitud de esta respuesta depende del tipo de ejercicio ${ }^{2}$. Además, se estima que entre 20 y $60 \%$ de la variación de la PA entre los individuos, es dado a factores genéticos ${ }^{3}$.

Uno de los principales reguladores de la PA es la enzima convertidora de angiotensina (ACE), un componente del sistema de renina angiotensina y aldosterona (RAAS), que media la conversión de la angiotensina I en angiotensina II (Ang II) y la degradación kininas, controla la vasoconstricción ${ }^{4}$.

A diferencia de la acción de la ACE, la ACE2 podría regular negativamente el RAAS mediante la generación de una vía vasodilatadora con generación de péptidos Ang 1-9 y Ang 1-75, que a su vez se une al receptor MAS6. Este eje ACE2 a través de su molécula Ang 1-7, ha demostrado 
Influencia de ACE2 en la respuesta hemodinámica aeróbica - N. Molina Gutiérrez et al

ser anti-hipertensiva que causa vasodilatación y mejora el metabolismo del músculo esquelético ${ }^{7,8}$.

El gen ACE2 está localizado en el cromosoma $\mathrm{X}^{9}$, comparte cerca de un $42 \%$ de semejanza con el dominio catalítico de la ACE somático. Se ha reportado que juega un papel protector en la regulación de la homeostasis de la presión arterial ${ }^{10,11}$. Esto se logra mediante la hidrolización de la apelina-36 en la apelina-13 por la ACE2, que también es responsable de la inactivación de la angiotensina I y $\mathrm{II}^{12,13}$. Los estudios de asociación entre los polimorfismos de un solo nucleótido (SNP, por sus siglas en inglés de Single Nucleotide Polymorphism) del ACE2 y la PA han arrojado diversos resultados en los seres humanos. En poblaciones australiana y alemana, los estudios no encontraron ninguna asociación entre los SNP del ACE2 y la PA ${ }^{14,15}$. Mientras que en población china el rs1978124 de este mismo gen, se asoció positivamente a niveles más altos de $\mathrm{PA}^{16}$. No obstante, cada población tiene distintas frecuencias en variantes genéticas, las que pueden matizar en mayor o menor medida el efecto de un solo polimorfismo. Por lo tanto, el objetivo de este estudio fue evaluar la influencia del polimorfismo rs1978124 del gen ACE2 en la respuesta hemodinámica post prueba aeróbica submáxima en jóvenes estudiantes chilenos sedentarios, con el fin de caracterizar algunas de las variables que pudiesen influir en la regulación de la presión arterial, que permitan explorar alternativas de tratamiento más precisas en sujetos que presentan un genotipo de riesgo.

\section{Materiales y Método}

\section{Sujetos}

El tamaño de la muestra se calculó utilizando el programa Epi-Info 7 (CDC, USA.), utilizando un intervalo de confianza de 95\%, un margen de error del $10 \%$ y una heterogeneidad de $50 \%$; lo que arrojó una muestra $\mathrm{N}$ de 96 individuos. Se seleccionó una muestra de 102 individuos chilenos: 47 hombres (21,51 $\pm 2,04$ años) y 55 mujeres $(21,71$ $\pm 2,45$ años), estudiantes de la Universidad Católica del Maule, que participaron voluntariamente formar parte del estudio. Los criterios de exclusión fueron el diagnóstico de hipertensión, diabetes, patologías musculo esqueléticas u osteoarticulares, uso de medicamentos que pudieran alterar los parámetros de interés, obesos, relación consan- guínea directa con otro participante, deportistas y personas que practican ejercicio al menos 3 veces por semana de manera regular. Todos los participantes firmaron el consentimiento informado aprobado por el Comité de Ética Científica de la Universidad Católica del Maule.

\section{Prueba submáxima aeróbica}

Como prueba aeróbica submáxima, se utilizó el protocolo de la Prueba de Caminata de 6 min de la Sociedad Torácica Americana (ATS) ${ }^{17}$. Los sujetos caminaban lo más rápido posible una distancia de 30 metros por un período de $6 \mathrm{~min}$. Al mismo tiempo, se estimulaba continuamente la marcha y se registraba la frecuencia cardíaca minuto a minuto, y la presión sanguínea se registraba antes e inmediatamente después de la prueba.

\section{Muestra de sangre y genotipado de ACE2}

Se extrajo ADN genómico de $5 \mathrm{cc}$ de sangre periférica mediante un procedimiento de $\mathrm{NaI}$ optimizado por Salazar et al. ${ }^{18}$. La genotipificación del ACE2 se realizó mediante la técnica PCR-RFLP, utilizando los partidores 5' GCCTCATGCTCTCTTCAC-3' y 3' CAGACCTCCAAGGACTCCA-5', diseñados con el software Primer3 v0.4.0 que está disponible en http://frodo.wi.mit.edu/. La reacción en cadena de la polimerasa (PCR) se realizó de acuerdo con el protocolo del fabricante (GoTAQ Promega, EE.UU.) en un termociclador T100 (Bio-Rad, EE.UU.) Las condiciones térmicas fueron: un paso de $95^{\circ} \mathrm{C}$ durante $5 \mathrm{~min}$, seguido de 32 ciclos de 30 segundos a $95^{\circ} \mathrm{C}, 60^{\circ} \mathrm{C}$ y $72^{\circ}$, y un paso final de $10 \mathrm{~min}$ a $72^{\circ} \mathrm{C}$. El producto de la PCR de $402 \mathrm{pb}$ fue digerido con la enzima ECO47I (Thermo Fisher Scientific, Inc.) bajo la recomendación del fabricante. Finalmente, los productos fueron sometidos a electroforesis en un gel de agarosa al 2\% y teñidos con GelRed ${ }^{\circ}$ (Biotium, Hayward, USA.). El alelo C fue interpretado con dos bandas de 309 y 93 pb. El alelo T fue una banda única de $402 \mathrm{pb}$. Dos investigadores independientes interpretaron la electroforesis del producto de restricción enzimática, para luego proceder a determinar los genotipos.

\section{Análisis estadístico}

Todos los análisis estadísticos se realizaron con el software GraphPad Prism 5.0 (GraphPad Software, EE.UU.). Los datos se presentaron como media \pm desviación estándar (DE). Se utilizó como 
método estadístico la prueba t no emparejada o ANOVA para comprobar las diferencias en la media entre los grupos según corresponda. La distribución del genotipo, la frecuencia de los alelos y el equilibrio de Hardy-Weinberg se probaron mediante un análisis de chi-cuadrado $\left(\chi^{2}\right)$. La significación estadística se fijó en $\mathrm{P}<0,05$.

\section{Resultados}

El análisis de los efectos del polimorfismo de la ACE2 se realizó por separado para los hombres y mujeres debido a que la variante genética se encuentra en el cromosoma X.

En el caso de las mujeres, la distribución del genotipo (Tabla 1) no se desvió de la predicha por el equilibrio de Hardy-Weinberg $\left(\chi^{2}=0,804242601\right.$, d.f. $=1, \mathrm{P}=0,369828)$. No hubo diferencias significativas en los parámetros biométricos entre los grupos de genotipo CC, CT y TT. La frecuencia cardíaca (FC), la presión sanguínea sistólica (PAS) y la presión sanguínea diastólica (PAD) tampoco tuvieron diferencias significativas después de la prueba aeróbica submáxima $(\mathrm{P}=0,098,0,6693$ y 0,924 respectivamente; ANOVA) (Tabla 2).

Para los hombres, el equilibrio de Hardy-Weinberg no pudo ser calculado debido a la herencia ligada al cromosoma $\mathrm{X}$ del polimorfismo. Los parámetros de la línea de base no presentaron diferencias significativas (Tabla 1). Tampoco hubo diferencias significativas en los cambios en FC, PAS y PAD entre los grupos de genotipo CC y TT después de la prueba aeróbica submáxima. ( $\mathrm{P}=0,835,0,621$ y 0,559 respectivamente; prueba t no emparejado) (Tabla 2).

Tabla 1. Características de base de la población estudiada estratificada por género

\begin{tabular}{|c|c|c|c|c|c|c|c|}
\hline \multirow[t]{2}{*}{ Características } & \multicolumn{3}{|c|}{ Hombres } & \multicolumn{4}{|c|}{ Mujeres } \\
\hline & $\begin{array}{c}\text { Genotipo } \\
\text { C }\end{array}$ & $\begin{array}{c}\text { Genotipo } \\
\mathbf{T}\end{array}$ & Valor $\mathbf{p}$ & $\begin{array}{c}\text { Genotipo } \\
\text { CC }\end{array}$ & $\begin{array}{c}\text { Genotipo } \\
\text { CT }\end{array}$ & $\begin{array}{c}\text { Genotipo } \\
\text { TT }\end{array}$ & Valor $\mathbf{p}$ \\
\hline Muestra, \% & $21(44,7)$ & $26(55,3)$ & & $8(14,5)$ & $30(54,5)$ & $17(30,9)$ & \\
\hline Edad, años & $22,2 \pm 2,2$ & $21,7 \pm 1,9$ & $0,4168^{b}$ & $21,50 \pm 3,2$ & $21,4 \pm 2,3$ & $22,2 \pm 2,2$ & $0,5045^{a}$ \\
\hline Peso, $\mathrm{Kg}$ & $71,1 \pm 10,5$ & $68,4 \pm 8,3$ & $0,3315^{b}$ & $56,2 \pm 6,9$ & $59,1 \pm 7,2$ & $57,3 \pm 4,8$ & $0,4395^{a}$ \\
\hline Talla, cms & $170,8 \pm 6,1$ & $170,9 \pm 6,6$ & $0,9276^{b}$ & $160,0 \pm 3,8$ & $158,7 \pm 5,5$ & $159,4 \pm 4,8$ & $0,7855^{a}$ \\
\hline
\end{tabular}

Los datos se reportan como media \pm DE o número de sujetos (\%). Test estadísticos: ${ }^{a} A N O V A$ unidireccional; Prueba de comparaciones múltiples; ${ }^{b}$ Prueba de t no emparejada * $\mathrm{P}<0,05$

Tabla 2. Resultados hemodinámicos en pruebas físicas según el polimorfismo del gen ECA2, análisis del genotipo, en hombres y mujeres

\begin{tabular}{|c|c|c|c|c|c|c|c|}
\hline \multirow[t]{2}{*}{ Características } & \multicolumn{3}{|c|}{ Hombres } & \multicolumn{4}{|c|}{ Mujeres } \\
\hline & $\begin{array}{c}\text { Genotipo } \\
\text { C }\end{array}$ & $\begin{array}{c}\text { Genotipo } \\
\mathbf{T}\end{array}$ & Valor p & $\begin{array}{c}\text { Genotipo } \\
\text { CC }\end{array}$ & $\begin{array}{c}\text { Genotipo } \\
\text { CT }\end{array}$ & $\begin{array}{c}\text { Genotipo } \\
\text { TT }\end{array}$ & Valor $\mathbf{p}$ \\
\hline FC Inicio,pulsos/min & $72,3 \pm 12,3$ & $72,2 \pm 11,1$ & $0,9851^{b}$ & $85,3 \pm 9,0$ & $86,5 \pm 13,4$ & $77,3 \pm 17,4$ & $0,1109^{a}$ \\
\hline FC Final, pulsos/min & $168,7 \pm 21,7$ & $167,2 \pm 27,0$ & $0,8354^{b}$ & $181,8 \pm 4,1$ & $184,7 \pm 13,3$ & $174,2 \pm 21,8$ & $0,0986^{a}$ \\
\hline PAS Inicio, mm Hg & $121,5 \pm 10,9$ & $122,6 \pm 10,8$ & $0,7044^{b}$ & $108,7 \pm 11,4$ & $117,23 \pm 9,2$ & $113,8 \pm 7,7$ & $0,0648^{a}$ \\
\hline PAD Final, mm Hg & $156,9 \pm 21,9$ & $154,1 \pm 15,8$ & $0,6214^{b}$ & $140,4 \pm 8,9$ & $136,3 \pm 13,5$ & $136,7 \pm 7,6$ & $0,6693^{a}$ \\
\hline DBP Inicio, mm Hg & $75,9 \pm 8,7$ & $77,2 \pm 8,9$ & $0,6646^{b}$ & $71,2 \pm 10,1$ & $75,6 \pm 7,3$ & $76,1 \pm 9,7$ & $0,3720^{a}$ \\
\hline PAD Final, mm Hg & $85,9 \pm 13,9$ & $88,1 \pm 11,8$ & $0,5597^{b}$ & $87,0 \pm 22,16$ & $86,7 \pm 11,5$ & $85,2 \pm 9,7$ & $0,9244^{a}$ \\
\hline
\end{tabular}

FC: Frecuencia Cardiaca; PAS: presión arterial sistólica; PAD: presión arterial diastólica. Los datos se reportan como media \pm DE. Prueba estadística: ${ }^{a} A N O V A$ unidireccional; Prueba de comparaciones múltiples; ${ }^{b}$ Prueba de t no emparejada *P $<0,05$. 


\section{Discusión}

En el presente estudio, evaluamos por primera vez un posible efecto del polimorfismo del ACE2 en la respuesta hemodinámica posterior a la prueba aeróbica submáxima en una muestra chilena sedentaria joven. Los principales hallazgos de este estudio muestran que no hubo diferencias previas ni posteriores las variables hemodinámicas como la FC, PAS y PAD en el grupo estudiado, en contraposición con las diferencias descritas en poblaciones orientales o caucásicas descritas por Patel, et al. ${ }^{19}$.

Malard et al., en un estudio de cohorte realizado a lo largo de 5 años, encontraron una asociación de diferentes SNPs del gen ACE2 con cambios en la presión sanguínea en los sujetos jóvenes ${ }^{20}$, sin embargo nuestro estudio intenta atribuir estos cambios a un estímulo agudo como la prueba aeróbica submáxima.

Cabe recalcar, que la variante genética descrita se encontraba en equilibrio Hardy-Weinberg para las mujeres, mientas que para los hombres no fue posible hacer este cálculo ya que esta variante se presenta en el cromosoma X, siendo un cromosoma único para el género y por lo tanto ofrece solo 2 alternativas, $\mathrm{C}$ o $\mathrm{T}$.

Los resultados obtenidos en este estudio se asemejan a los encontrados en población adolescentes franco-canadienses ${ }^{20}$, australianos ${ }^{14} y$ chinos $^{21}$, lo que podría indicar que el polimorfismo (rs1978124) de la ACE2 no tiene un impacto importante en los cambios de la presión sanguínea en sujetos sedentarios en contraposición con otros polimorfismos del mismo gen ${ }^{15,22}$. Sin embargo, la mayoría de esos estudios analizan las variables hemodinámicas en sujetos con algún tipo de patología cardiovascular ${ }^{16,21,23}$, lo que significa que el sistema ya se encuentra expuesto a una condición de estrés basal. Este último factor, pudiese acentuar el papel que juega la ACE y ACE2 en la modulación de la respuesta hemodinámica frente a un estímulo, evidenciando diferencias en variables como la presión arterial.

Con esto en cuenta, y dado que se ha demostrado en modelos animales que el entrenamiento físico promueve cambios en la expresión del ACE2 y que la expresión del gen de la ACE2 se presenta como un potente vasodilatador ${ }^{24}$, sería interesante continuar este estudio buscando la asociación de esta variante genética y la respuesta del sistema cardiocirculatorio frente a un estímulo aeróbico máximo.

En conclusión, nuestros resultados indican que el polimorfismo rs1978124 del gen ACE2, no influye en la respuesta hemodinámica en estudiantes chilenos después de la prueba aeróbica submáxima.

Agradecimientos: Los investigadores quieren agradecer a los estudiantes de la Escuela de Kinesiología de la Universidad Católica del Maule por su participación en este estudio.

\section{Referencias}

1. Gronek P, Holdys J. Genes and physical fitness. Trends Sport Sci. 2013; 20 (1): 16-29.

2. Fagard RH. Exercise characteristics and the blood pressure response to dynamic physical training. In: Medicine and Science in Sports and Exercise 2001.

3. Kurtz TW, Spence MA. Genetics of essential hypertension. Am J Med [Internet]. 1993 Jan [cited 2020 Mar 2]; 94 (1): 77-84. Available from: http://www.ncbi.nlm.nih. gov/pubmed/8420305

4. Coates D. The angiotensin converting enzyme (ACE). Int J Biochem Cell Biol [Internet]. 2003 Jun [cited 2020 Mar 2]; 35 (6): 769-73. Available from: http://www.ncbi. nlm.nih.gov/pubmed/12676162

5. Eriksson U, Danilczyk U, Penninger JM. Just the beginning: Novel functions for angiotensin-converting enzymes. Current Biology 2002; Vol 12.

6. Santos RAS, Simoes e Silva AC, Maric C, Silva DMR, Machado RP, De Buhr I, et al. Angiotensin-(1-7) is an endogenous ligand for the $G$ protein-coupled receptor Mas. Proc Natl Acad Sci U S A. 2003 Jul 8; 100 (14): 8258-63.

7. Prasannarong M, Santos FR, Henriksen EJ. ANG-(1-7) reduces ANG II-induced insulin resistance by enhancing Akt phosphorylation via a Mas receptor-dependent mechanism in rat skeletal muscle. Biochem Biophys Res Commun [Internet]. 2012 Sep 28 [cited 2020 Mar 2]; 426 (3): 369-73. Available from: http://www.ncbi.nlm. nih.gov/pubmed/22960175

8. Ocaranza Dr MP, Michea L, Chiong M, Lagos CF, Lavandero S, Jalil JE. Recent insights and therapeutic perspectives of angiotensin-(1-9) in the cardiovascular system [Internet]. Clinical Science. Portland Press Ltd 2014 [cited 2020 Feb 28]; Vol 127: 549-57. Available from: https://portlandpress.com/clinsci/article/127/9/549/70762/ Recent-insights-and-therapeutic-perspectives-of 
9. Yang M, Zhao J, Xing L, Shi L. The association between angiotensinconverting enzyme 2 polymorphisms and essential hypertension risk: A metaanalysis involving 14,122 patients. JRAAS - J Renin-Angiotensin-Aldosterone Syst. 2015; 16 (4): 1240-4.

10. Tipnis SR, Hooper NM, Hyde R, Karran E, Christie G, Turner AJ. A human homolog of angiotensin-converting enzyme. Cloning and functional expression as a captopril-insensitive carboxypeptidase. J Biol Chem [Internet] 2000 Oct 27 [cited 2020 Mar 2]; 275 (43): 33238-43. Available from: http://www.ncbi.nlm.nih.gov/ pubmed/10924499

11. Yagil Y, Yagil C. Hypothesis: ACE2 modulates blood pressure in the mammalian organism. Hypertens (Dallas, Tex 1979) [Internet] 2003 Apr 1 [cited 2020 Mar 2]; 41 (4): 871-3. Available from: http://www.ncbi.nlm. nih.gov/pubmed/12654716

12. Vickers C, Hales P, Kaushik V, Dick L, Gavin J, Tang J, et al. Hydrolysis of biological peptides by human angiotensin-converting enzyme-related carboxypeptidase. J Biol Chem. 2002 Apr 26; 277 (17): 14838-43.

13. Burrell LM, Johnston CI, Tikellis C, Cooper ME. ACE2, a new regulator of the renin-angiotensin system [Internet]. Trends in Endocrinology and Metabolism. 2004 [cited 2020 Mar 2]; Vol 15: 166-9. Available from: http://www.ncbi.nlm.nih.gov/pubmed/15109615

14. Benjafield AV, Wang WYS, Morris BJ. No association of angiotensin-converting enzyme 2 gene (ACE2) polymorphisms with essential hypertension. Am J Hypertens [Internet]. 2004 Jul [cited 2020 Mar 2]; 17 (7): 624-8. Available from: http://www.ncbi.nlm.nih.gov/pubmed/15233982

15. Lieb W, Graf J, Götz A, König IR, Mayer B, Fischer M, et al. Association of angiotensin-converting enzyme 2 (ACE2) gene polymorphisms with parameters of left ventricular hypertrophy in men: Results of the MONICA Augsburg echocardiographic substudy. J Mol Med. 2006; 84 (1): 88-96.

16. Niu W, Qi Y, Hou S, Zhou W, Qiu C. Correlation of angiotensin-converting enzyme 2 gene polymorphisms with stage 2 hypertension in Han Chinese. Transl Res.
2007; 150 (6): 374-80.

17. Crapo RO, Casaburi R, Coates AL, Enright PL, MacIntyre NR, McKay RT, et al. ATS statement: Guidelines for the six-minute walk test. American Journal of Respiratory and Critical Care Medicine. American Lung Association 2002; Vol 166: 111-7.

18. Salazar L, Melo C, Cavalli S, Hinuy H, Hirata M, Hirata RD. Micrométodo para extraçäo de DNA genômico útil no diagnóstico molecular da Hipercolesterolemia Familial. Rev bras anal clin. 2001; 33 (3): 111-6.

19. Patel SK, Velkoska E, Freeman M, Wai B, Lancefield TF, Burrell LM. From gene to protein-experimental and clinical studies of ACE2 in blood pressure control and arterial hypertension. Front Physiol. 2014; 5 JUN (June): $1-12$.

20. LM, LK, JO, M-H R-G, AL, LP, et al. Association between the angiotensin-converting enzyme-2 gene and blood pressure in a cohort of canadian adolescents. Circulation [Internet]. 2012; 125 (10 SUPPL. 1). Available from: http://ovidsp.ovid.com/ovidweb.cgi?T=JS\&PA$\mathrm{GE}=$ reference $\& \mathrm{D}=$ emed $11 \& \mathrm{NEWS}=\mathrm{N} \& \mathrm{AN}=70950895$

21. Liu D, Chen Y, Zhang P, Zhong J, Jin L, Zhang C, et al. Association between circulating levels of ACE2-Ang-(17)-MAS axis and ACE2 gene polymorphisms in hypertensive patients. Med (United States). 2016; 95 (24): 1-6.

22. Huang W, Yang W, Wang Y, Zhao Q, Gu D, Chen R. Association study of angiotensin-converting enzyme 2 gene (ACE2) polymorphisms and essential hypertension in northern Han Chinese. J Hum Hypertens [Internet] 2006 Dec [cited 2020 Mar 2]; 20 (12): 968-71. Available from: http://www.ncbi.nlm.nih.gov/pubmed/17024138

23. Goessler KF, Polito MD, Mota G de F, de Oliveira EM, Cornelissen VA. Angiotensin converting enzyme 2 polymorphisms and postexercise hypotension in hypertensive medicated individuals. Clin Physiol Funct Imaging. 2018; 38 (2): 206-12.

24. Gomes-Santos IL, Fernandes T, Couto GK, Ferreira-Filho JCA, Salemi VMC, Fernandes FB, et al. Effects of exercise training on circulating and skeletal muscle renin-angiotensin system in chronic heart failure rats. PLoS One. 2014; 9 (5): 1-11. 